\title{
Acquisition of paclitaxel resistance via PI3K-dependent epithelial-mesenchymal transition in A2780 human ovarian cancer cells
}

\author{
FANGFANG DU $^{1 *}$, XIAOHONG WU ${ }^{2 *}$, YANJUN LIU $^{1}$, TENG WANG $^{2}$, XIAOWEI QI ${ }^{2}$, YONG MAO $^{2}$, LI JIANG $^{1}$, \\ YIFEI ZHU ${ }^{1}$, YUN CHEN ${ }^{1}$, RUIYU ZHU ${ }^{1,4}$, XIAOFENG HAN $^{3}$, JIAN JIN $^{1,4}$, XIN MA $^{1,4}$ and DONG HUA ${ }^{2}$ \\ ${ }^{1}$ School of Pharmaceutical Sciences, ${ }^{2}$ Affiliated Hospital and ${ }^{3}$ School of Medicine, Jiangnan University, \\ Wuxi, Jiangsu; ${ }^{4}$ Institute of Health Sciences, Shanghai Institutes for Biological Sciences, \\ Chinese Academy of Sciences, Shanghai, P.R. China
}

Received February 12, 2013; Accepted April 10, 2013

DOI: 10.3892/or.2013.2567

\begin{abstract}
Epithelial ovarian cancer is a major cause of mortality among women with gynecological malignancies. Paclitaxel is commonly used for chemotherapy of ovarian cancer, yet its efficacy is limited by chemoresistance. Generally, drug resistance is associated with acquisition of the epithelial-mesenchymal transition (EMT) in cancer. The aim of the present study was to determine whether the EMT is involved in acquired resistance to paclitaxel in A2780 human ovarian cancer cells. Using the paclitaxel-resistant A2780/PTX cell line, we examined the cellular morphology, molecular changes, migration and proliferation consistent with the EMT. Furthermore, we found that inhibition of phosphatidylinositol 3-kinase (PI3K) activity reduced the proliferation and migration and restored their sensitivity to paclitaxel. Our study provides new insights into EMT-like phenotypic changes that are linked to paclitaxel resistance in A2780 cells. We believe that inhibition of the PI3K signaling pathway could provide a novel therapeutic approach to overcome chemoresistance and prevent metastasis during paclitaxel chemotherapy.
\end{abstract}

\section{Introduction}

Epithelial ovarian cancer is a major cause of mortality among women with gynecological malignancies due to its late symp-

Correspondence to: Dr Dong Hua, Affiliated Hospital, Jiangnan University, 200 Huihe Road, Wuxi, Jiangsu 214062, P.R. China

E-mail: huadong@csco.org.cn

Professor Xin Ma, School of Pharmaceutical Sciences, Jiangnan University, 1800 Lihu Road, Wuxi, Jiangsu 214122, P.R. China

E-mail: maxin@jiangnan.edu.cn

${ }^{*}$ Contributed equally

Key words: ovarian cancer, epithelial-to-mesenchymal transition, paclitaxel, chemoresistance tomatology (1); it is often associated with distant metastases and has a poor prognosis (2). Chemotherapy is essential for the management of ovarian cancer, but the efficacy of this treatment is limited by chemoresistance. Paclitaxel is a first-line chemotherapeutic agent that is effective in epithelial ovarian cancer by stabilizing microtubules, inducing cell cycle arrest in the G2-M phase, and activating pro-apoptotic signaling $(2,3)$. In spite of the comparatively high sensitivity of epithelial ovarian cancer to paclitaxel, the prognosis of advanced or recurrent cases remains poor since mortality is mostly the result of metastasis that is refractory to conventional chemotherapy (2).

The epithelial-mesenchymal transition (EMT) in cancer cells has been shown to promote metastasis and chemoresistance (4). During the acquisition of EMT characteristics, the hallmark of EMT is loss of the epithelial molecule E-cadherin and gain of mesenchymal markers including $\mathrm{N}$-cadherin and vimentin, leading to remodeling of the cytoskeleton and enhancement of cancer cell migration and invasion (5). An EMT phenotype has been reported in gemcitabine-resistant pancreatic cancer cells (6), gefitinib-resistant non-small cell lung cancer and oxaliplatin-resistant colorectal cancer cells (7), paclitaxel-resistant ovarian cancer cells (2) and tamoxifenresistant breast cancer cells (8). Blockade of the EMT pathways is critical for preventing cancer cell migration and invasion as well as for restoring drug sensitivity (4). Although the EMT in cancer cells integrates multiple signaling pathways, such as TGF $\beta$, NF- $\kappa$ B, Wnt and Hedgehog (9-12), the details in A2780 human ovarian cancer cells remain to be identified.

We are the first to report that phosphatidylinositol 3-kinase (PI3K) inhibition reverses paclitaxel resistance-induced EMT in A2780 human ovarian cancer cells, indicating that the PI3K pathway is a promising therapeutic target against ovarian cancer with paclitaxel chemotherapy.

\section{Materials and methods}

Cells and reagents. A2780/WT and A2780/PTX cell lines were obtained from KeyGen Biotech Co. Ltd. (Nanjing, China) and maintained in RPMI-1640 supplemented with $10 \%$ fetal bovine serum (FBS) and penicillin-streptomycin 
$\left(100 \mathrm{U} / \mathrm{ml}\right.$ penicillin and $100 \mu \mathrm{g} / \mathrm{ml}$ streptomycin) at $37^{\circ} \mathrm{C}$ in a humidified atmosphere of $5 \% \mathrm{CO}_{2}$. Paclitaxel was from Qilu Pharmaceutical Co, Ltd. (Jinan, China). LY294002 was from Selleckchem (Burlington, NC, USA). The primers were synthesized by Sangon Biotechnology (Shanghai, China).

Reverse transcription (RT)-PCR. Total RNA was extracted using the TRIzol protocol and cDNA was synthesized from the mRNA using the SuperScript First-Strand Synthesis System (Invitrogen Life Technologies, Carlsbad, CA, USA) for RT-PCR. PCR was performed for 35 cycles of $95^{\circ} \mathrm{C}$ for $30 \mathrm{sec}, 58^{\circ} \mathrm{C}$ for $30 \mathrm{sec}$ and $72^{\circ} \mathrm{C}$ for $45 \mathrm{sec}$. The data were analyzed with ImageJ software (NIH, Bethesda, MD, USA). $\beta$-actin was used as endogenous control. The primer sequences are available upon request.

Migration assay. Cell migration was studied by the modified Boyden chamber method in 24-well plates, each containing 6.5-mm Transwell chambers with $8-\mathrm{mm}$ pores (BD Biosciences, Franklin Lakes, NJ, USA). Cells ( $1 \times 10^{6}$ cells $\left./ \mathrm{ml}\right)$ were harvested, washed twice with PBS and responded in serum-free RPMI-1640. The cell suspension $(100 \mu \mathrm{l})$ was seeded into the upper chamber of each Transwell unit. The bottom wells contained RPMI-1640 supplemented with $10 \%$ FBS to create a chemotactic gradient. During certain treatments, PI3K inhibitor LY294002 $(10 \mu \mathrm{M})$ was added. After $24 \mathrm{~h}$ of stimulation, the cells that had not migrated through the filter in the Transwell inserts (on the upper surface) were removed with a cotton swab. Cells that had migrated to the lower surface of the filter were stained with crystal violet and counted under a microscope. Images were captured by a video camera (Nikon Coolpix 54, Japan) mounted on the microscope (Leica CME, Japan). Cells were counted in four random fields per insert.

Wound-healing assay. Cell motility was assessed by measuring the ability of the cells to migrate into a wound made in a confluent monolayer. Briefly, wild-type A2780/WT and A2780/PTX cells were plated at $2 \times 10^{5}$ cells/well in 6-well plates. After overnight growth and attachment, both media were replaced with serum-free media for another $24 \mathrm{~h}$. The monolayers were scratched using a sterile pipette tip; floating cells were washed off with PBS. The cells were incubated in $2 \%$ FBS medium with or without LY294002 $(10 \mu \mathrm{M})$, The ability of cells to migrate into the wound gap was observed and recorded photographically. Wound closure was analyzed as the ratio of the remaining wound area relative to the initial wound area with ImageJ software (NIH). Experiments were repeated at least 3 times.

In vitro cell proliferation assay. Serial dilutions of cells in culture medium were prepared, and $100 \mu \mathrm{l}$ of the suspensions containing $1 \times 10^{4}, 5 \times 10^{3}$ and $1 \times 10^{3}$ cells were each added into 6-wells of 96-well plates. Cells were incubated for $48 \mathrm{~h}$, then $10 \mu \mathrm{l}$ MTT (5 mg/ml) (Sigma-Aldrich, St. Louis, MO, USA) was added to form formazan crystals, which were dissolved in $150 \mu 1$ dimethyl sulfoxide (DMSO). Absorbance was measured at $490 \mathrm{~nm}$ using a plate reader (Thermo Labsystems Multiskan MK3; Thermo Scientific, Rockford, IL, USA). The values from triplicate readings were averaged.
Paclitaxel chemosensitivity assay. The cells were seeded at 8,000 cells/well (triplicates) in 96-well plates. After $24 \mathrm{~h}$, they were treated with paclitaxel $(1.5-1,500 \mu \mathrm{g} / \mathrm{ml}$ in 2-fold serial dilutions) for $48 \mathrm{~h}$. After the treatment, MTS (20 $\mu \mathrm{l}$; Promega Corporation, Madison, WI, USA) was added to each well and the cells were incubated at $37^{\circ} \mathrm{C}$ with $5 \% \mathrm{CO}_{2}$ for $4 \mathrm{~h}$. Absorbance at $590 \mathrm{~nm}$ was then measured with a microplate reader (Thermo Synergie HT, USA).

Immunofluorescence staining. The cells were fixed for $30 \mathrm{~min}$ in $4 \%$ paraformaldehyde, permeabilized with $0.1 \%$ Triton X-100 for $10 \mathrm{~min}$ at room temperature, respectively. Cells were washed 3 times in PBS after each treatment. They were then blocked with $2 \%$ BSA in PBS for 30 min at room temperature, incubated with primary antibody for $\beta$-tubulin $(20 \mu \mathrm{g} / \mathrm{ml})$ diluted in $2 \% \mathrm{BSA} / \mathrm{PBS}$ overnight at $4^{\circ} \mathrm{C}$, and then incubated with fluorescent secondary antibody for $1 \mathrm{~h}$ at room temperature. Images were acquired with a confocal laser scanning microscope (LSM 710; Carl Zeiss, Jena, Germany). If required, cells were pretreated with LY294002 $(10 \mu \mathrm{M}, 24 \mathrm{~h})$.

Statistical analysis. Results are presented as the means \pm SD. Statistical differences were determined by Student's t-test. A value of $p<0.05$ was considered to indicate a statistically significant difference.

\section{Results}

Acquisition of paclitaxel resistance in A2780/PTX cells induces morphological and molecular changes consistent with EMT signaling. We first examined the morphological characteristics of the cell lines during exponential growth. A2780/WT cells formed cohesive clusters with round cellular morphology in vitro (Fig. 1A), consistent with an epithelial phenotype. By contrast, A2780/PTX cells, grown in the presence of $100 \mathrm{nM}$ PTX, displayed a spindle-like morphology and formed dyscohesive sheets (Fig. 1A).

To determine whether the acquisition of paclitaxel resistance induced specific molecular changes consistent with EMT, we investigated EMT-related biomarkers. Compared with the wild-type cells, the A2780/PTX cells showed significant reduction in E-cadherin and laminin-5 expression and upregulation of N-cadherin, vimentin and Twist (Fig. 1B).

A2780/PTX cells display increased potential for proliferation and migration. We performed wound healing and Transwell migration assays to compare the migratory potential between the 2 cell lines. The capacity for wound healing in A2780/PTX cells was significantly enhanced (Fig. 2A), and the relative numbers of migrating and invasive A2780/PTX cells were significantly superior to wild-type cells (Fig. 2B).

We further investigated the proliferative potential of A2780/WT and A2780/PTX cells at various times after plating and with various cell numbers at $48 \mathrm{~h}$ by assessing cell viability using a modified MTT assay. The MTT assay with various numbers showed a decrease in the growth of A2780/WT cells compared with A2780/PTX cells. In addition, similar results were found in the proliferative potential at various times (Fig. 2C). 
A

B
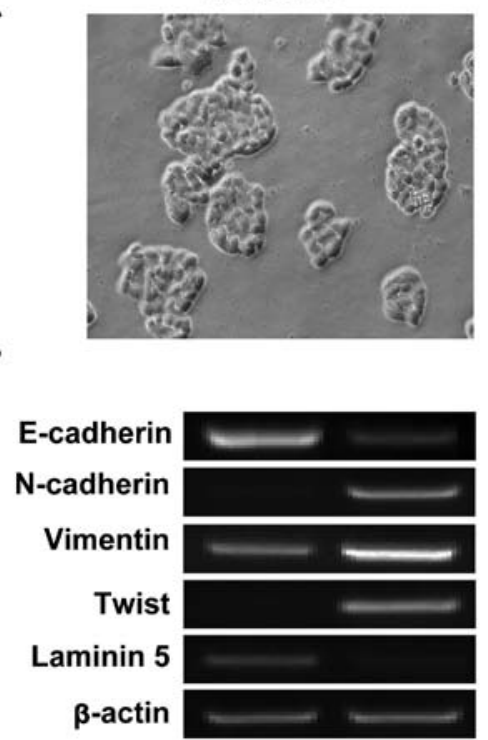

A2780/PTX
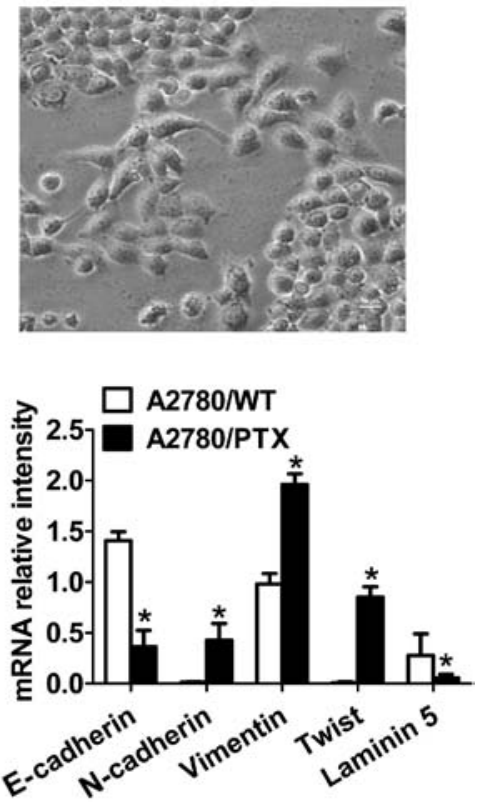

Figure 1. Acquisition of paclitaxel resistance in A2780/PTX cells induces morphologic and molecular changes consistent with the epithelial-mesenchymal transition (EMT) phenotype. (A) A2780/WT cells displayed a cobblestone appearance and growth in clusters (left); A2780/PTX cells lost adhesiveness and showed a spindle-shaped morphology (right). Cells were examined using phase-contrast microscopy. (B) Representative images (left) and summary (right) show EMT-related biomarkers expressed in A2780/PTX cells by RT-PCR. * $<<0.05$ compared with A2780/WT cells.

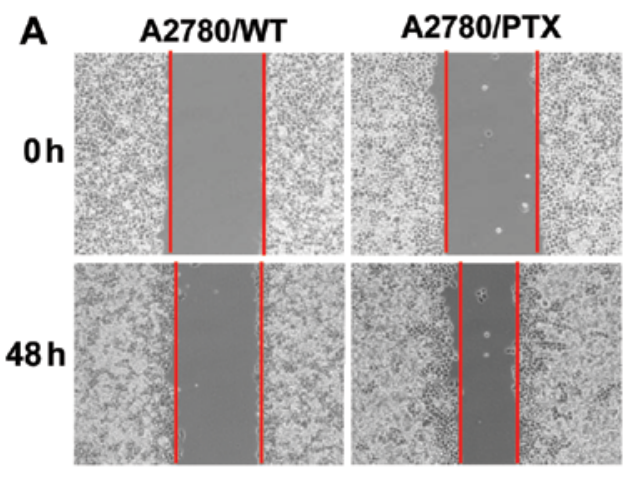

\section{B}

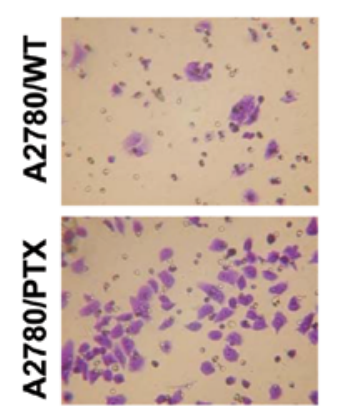

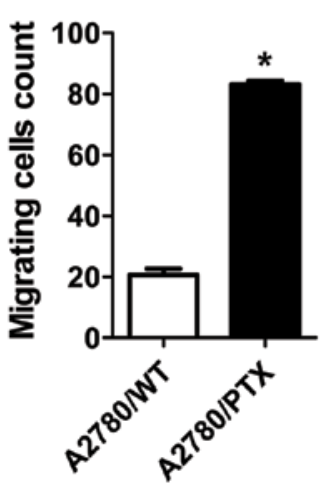

C
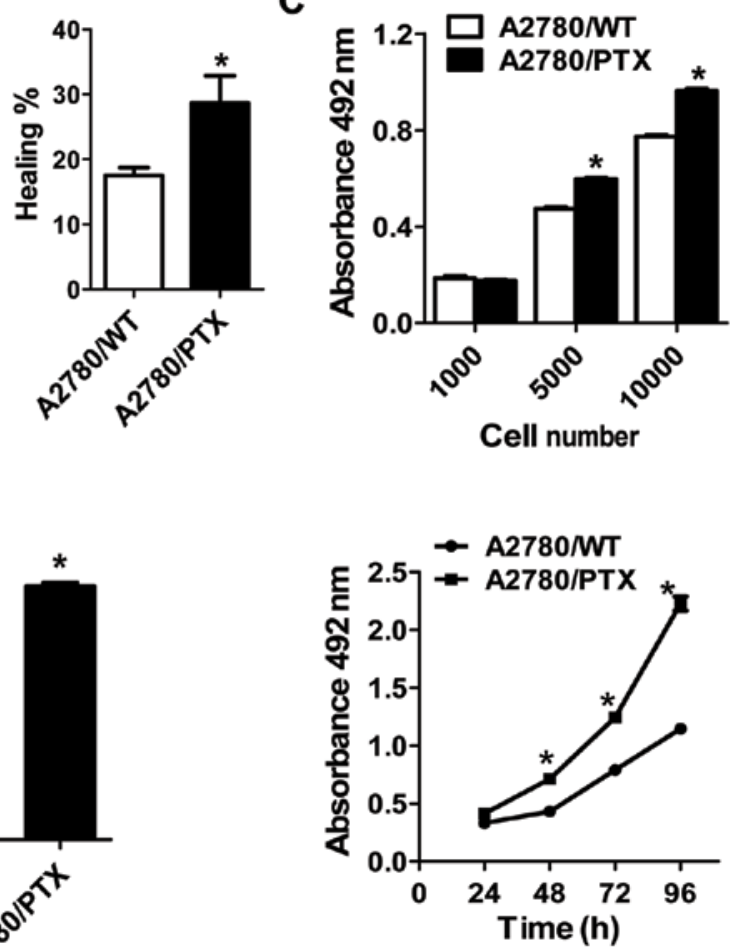

Figure 2. A2780/PTX cells display increased potential for proliferation and migration. (A) Wound-scratch assays showed increases in migration at $48 \mathrm{~h}$ in A2780/PTX cells. (B) Transwell chamber insert assays showed increase in migration (left) and quantitative results are shown as migrating cell count (right). (C) A2780/PTX cells exhibited an increase in growth at various numbers at $48 \mathrm{~h}$ after plating (up) or at various times (low) compared with A2780/WT cells. * $\mathrm{p}<0.05$ compared with A2780/WT cells.

PI3K signaling pathway is important for the induction of cell migration and proliferation. Recently, activation of the PI3K/AKT axis has been shown to be a central feature of
EMT (13-16). To assess the involvement of PI3K signaling, the PI3K inhibitor LY294002 $(10 \mu \mathrm{M})$ was applied to A2780/PTX cells, and they exhibited well-organized cell- 
A A2780/PTX
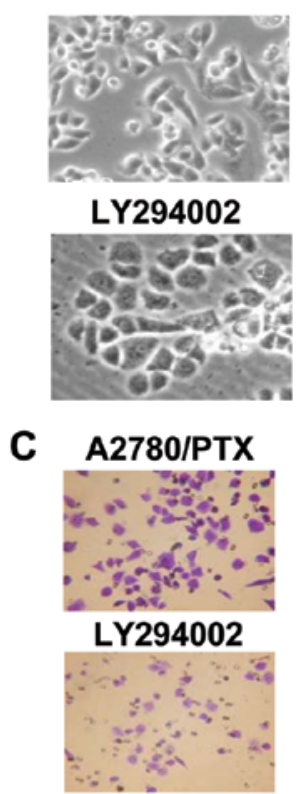

$\mathbf{E}$

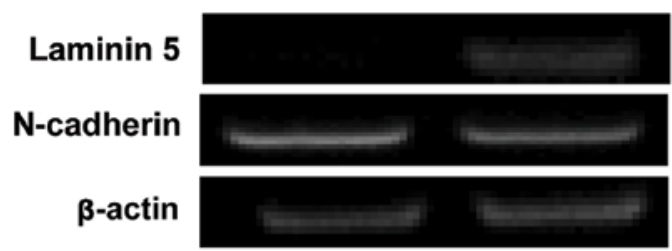

B A2780/PTX LY294002
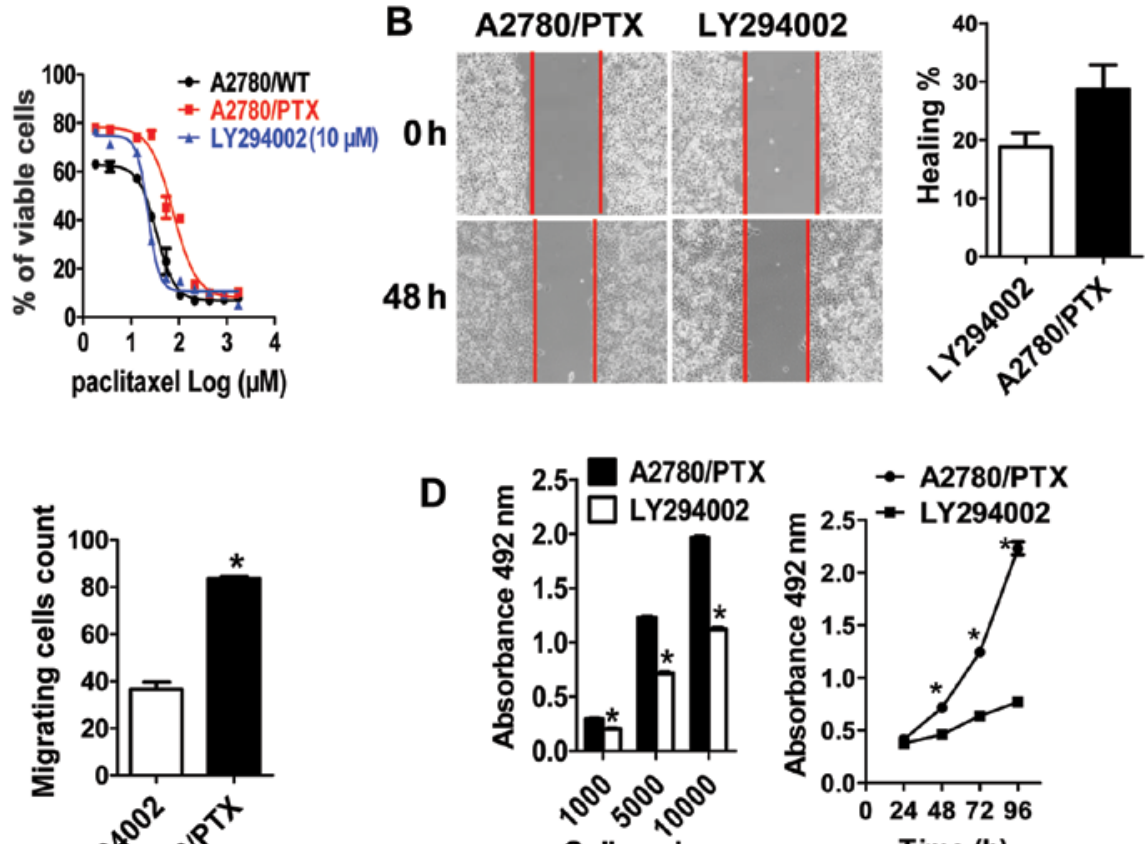

Cell number
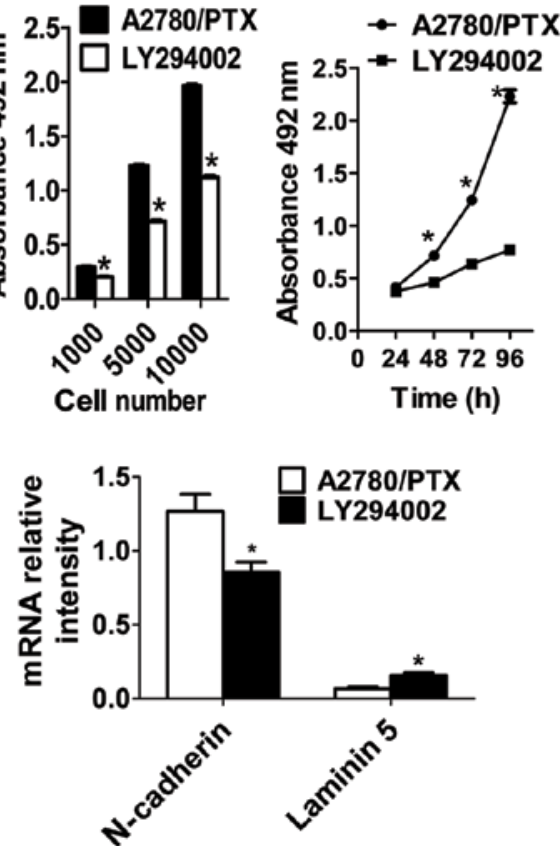

Figure 3. Inhibition of PI3K activity reverses paclitaxel-induced epithelial-mesenchymal transition (EMT) and increases sensitivity of A2780/PTX cells to paclitaxel. (A) Inhibition of PI3K reversed the EMT phenotype and increased sensitivity to paclitaxel in A2780/PTX cells. (B) Wound healing assay showed an inhibitory effect on migration by LY294002. (C) Inhibition of migration by Transwell assays. (D) Cell viability assays showed that treatment with LY294002 decreased proliferation in A2780/PTX cells. (E) Representative images (left) and summary data (right) show EMT-related biomarkers expressed in A2780/ PTX cells were partly reversed by LY294002 $(10 \mu \mathrm{M})$ by RT-PCR. " $<<0.05$ compared to A2780/PTX cells without LY294002.

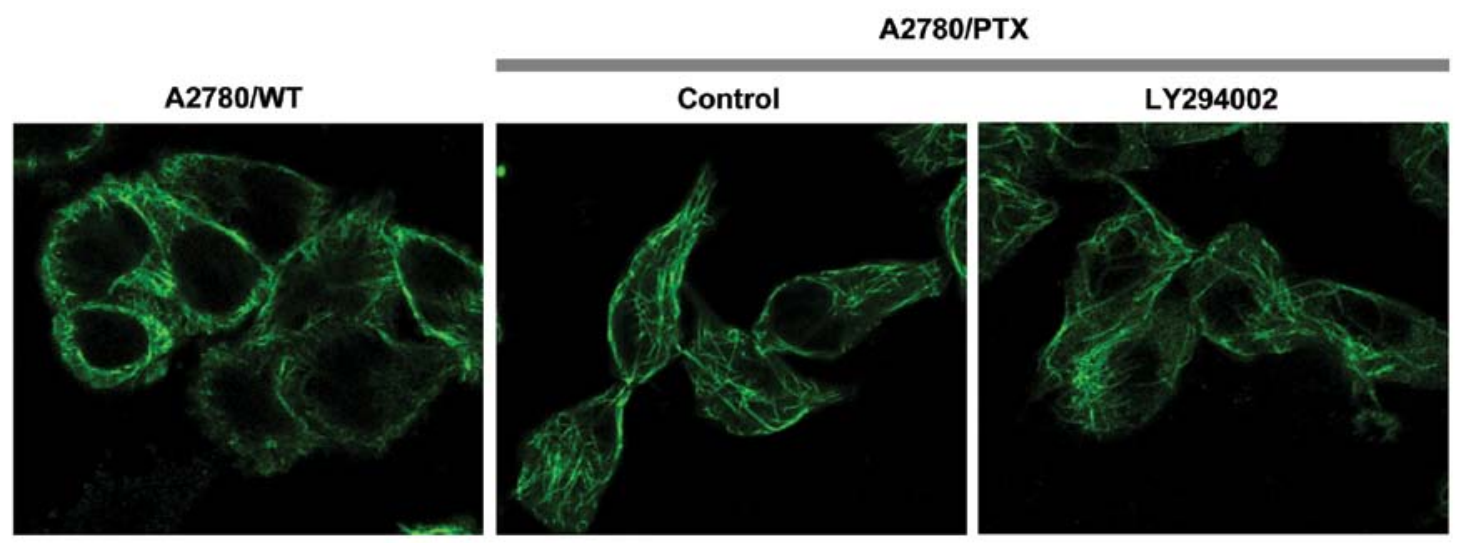

Figure 4. Inhibition of the PI3K activity alters the cytoskeleton of A2780/PTX cells. Representative cytoskeletal rearrangements ( $\beta$-tubulin, green) treated by LY294002 $(10 \mu \mathrm{M})$ in A2780/PTX cells. Control indicates dimethyl sulfoxide (DMSO) (0.1\%).

cell association, appeared cohesive, and exhibited classical epithelial morphology (Fig. 3A). The expression of epithelial marker laminin 5 was significantly enhanced, while the levels of mesenchymal marker N-cadherin decreased (Fig. 3E). Inhibition of PI3K significantly decreased migration and proliferation in A2780/PTX cells (Fig. 3B-D). These results 
showed that inhibition of PI3K signaling in A2780/PTX cells led to reversal of the EMT, indicating the involvement of the PI3K signaling pathway in the chemoresistance process.

Inhibition of the PI3K activity increases sensitivity of A2780/PTX cells to paclitaxel. We next explored whether inhibiting PI3K reverses the resistance to paclitaxel in A2780/ PTX cells. MTT assays showed that A2780/PTX cells treated with LY294002 restored the sensitivity to paclitaxel-induced cell death (Fig. 3A). The $\mathrm{IC}_{50}$ was reduced to half of that in untreated A2780/PTX cells.

Inhibition of the PI3K activity alters the cytoskeleton of A2780/PTX cells. The anticancer drug paclitaxel binds to $\beta$-tubulin in assembled microtubules and causes cytoskeleton changes in cells $(17,18)$. We further investigated organization of the cytoskeleton in A2780/PTX cells. A2780/PTX cells had spindle-like morphology or filopodia formation, in which brightly stained longitudinal $\beta$-tubulin bundles were detected, whereas cells in the wild-type and LY294002-treated groups displayed polygonal cell shapes (Fig. 4).

\section{Discussion}

Chemoresistance remains the major therapeutic barrier in epithelial ovarian cancer $(4,19)$. A thorough understanding of the mechanisms is essential for improving therapeutic agents. Our study used wild-type (A2780/WT) and paclitaxel-resistant human epithelial ovarian cancer cells (A2780/PTX) to investigate the molecular mechanism of paclitaxel resistance and associated cellular behaviors.

In the present study, we demonstrated that A2780/PTX cells underwent EMT processes. This was confirmed as follows: (i) morphological change from cobblestone-like to spindle-shaped cells; (ii) increased potential for proliferation and migration; and (iii) changes in molecular markers (significant reduction in E-cadherin and laminin-5 expression and upregulation of $\mathrm{N}$-cadherin and vimentin). In agreement with our results, some reports have shown that the EMT is induced by other chemotherapeutic agents in different cancer cell lines (20-22). Moreover, several studies have demonstrated that the EMT plays an important role in functional malignancy behaviors including facilitating the transformation, initiation, progression and metastasis of human cancer $(23,24)$. We further examined the proliferation in these 2 cell types. As expected, our findings showed that A2780/PTX cells displayed enhanced multiplication in the MTT assay. Overall, we obtained evidence that paclitaxel resistance and facilitated malignant potential are linked with the EMT.

Since the PI3K/AKT axis is frequently activated in human cancer (15), we focused on the EMT with particular emphasis on the PI3K/AKT pathways involved in the regulation of malignancy. To determine whether the PI3K pathway was involved in the process, the PI3K inhibitor LY294002 was applied to A2780/PTX cells, resulting in inhibition of fibroblastic morphology, proliferation and motility. Our evidence seems to be associated with other research in gefitinib-resistant cells from a head and neck squamous cell carcinoma cell line (22). Furthermore, we found that the inhibition of PI3K activity attenuated the paclitaxel resistance and led to loss of cell polarity in terms of $\beta$-tubulin distribution suggesting that there should also be defects in cell migration and invasion. Although further studies are required to elucidate the chemoresistance mechanisms in PI3K-mediated EMT, the use of PI3K inhibitors during chemotherapy could be a useful approach to improve its efficacy.

In conclusion, we have shown that the development of paclitaxel resistance in epithelial ovarian cancer cells is accompanied by inducible EMT-like changes with increased potential to metastasize. Targeting with the PI3K inhibitor LY294002 may sensitize cancer cells to chemotherapeutics and reduce motility. The clinical significance of the PI3K signal in acquired resistance to paclitaxel merits further investigation.

\section{Acknowledgements}

This study was supported by the Program for New Century Excellent Talents in University of The Ministry of Education of China (to X.M.); Fundamental Research Funds for the Central Universities JUSRP51311A (to X.M.); China National Natural Science Foundation grants 81100185 (to X.M.), 81130057 (to J.J.), 81201600 (to D.H.) and 81101667 (to R.Z.); Jiangsu Province National Natural Science Foundation grant BK2010161 (to D.H.); and a 'Strategic Priority Research Program' grant from the Chinese Academy of Sciences XDA01040000 (to J.J.).

\section{References}

1. Vergara D, Merlot B, Lucot JP, et al: Epithelial-mesenchymal transition in ovarian cancer. Cancer Lett 291: 59-66, 2010.

2. Kajiyama H, Shibata K, Terauchi M, et al: Chemoresistance to paclitaxel induces epithelial-mesenchymal transition and enhances metastatic potential for epithelial ovarian carcinoma cells. Int J Oncol 31: 277-283, 2007.

3. Jia L, Zhang S, Ye Y, et al: Paclitaxel inhibits ovarian tumor growth by inducing epithelial cancer cells to benign fibroblastlike cells. Cancer Lett 326: 176-182, 2012.

4. Rosano L, Cianfrocca R, Spinella F, et al: Acquisition of chemoresistance and EMT phenotype is linked with activation of the endothelin A receptor pathway in ovarian carcinoma cells. Clin Cancer Res 17: 2350-2360, 2011.

5. Thiery JP: Epithelial-mesenchymal transitions in tumour progression. Nat Rev Cancer 2: 442-454, 2002.

6. Shah AN, Summy JM, Zhang J, Park SI, Parikh NU and Gallick GE: Development and characterization of gemcitabineresistant pancreatic tumor cells. Ann Surg Oncol 14: 3629-3637, 2007.

7. Rho JK, Choi YJ, Lee JK, et al: Epithelial to mesenchymal transition derived from repeated exposure to gefitinib determines the sensitivity to EGFR inhibitors in A549, a non-small cell lung cancer cell line. Lung Cancer 63: 219-226, 2009.

8. Hiscox S, Jiang WG, Obermeier K, et al: Tamoxifen resistance in MCF7 cells promotes EMT-like behaviour and involves modulation of $\beta$-catenin phosphorylation. Int J Cancer 118: 290-301, 2006.

9. Zhou Q, Zeng R, Xu C, et al: Erbin inhibits TGF- $\beta 1$-induced EMT in renal tubular epithelial cells through an ERK-dependent pathway. J Mol Med (Berl) 90: 563-574, 2012.

10. Conti B, Minutolo A, Arciello M and Balsano C: Are Hedgehog and Wnt $/ \beta$-catenin pathways involved in hepatitis $C$ virusmediated EMT? J Hepatol 58: 636-637, 2012.

11. Radisky DC and Bissell MJ: NF- $\mathrm{BB}$ links oestrogen receptor signalling and EMT. Nat Cell Biol 9: 361-363, 2007.

12. Xu X, Zhou Y, Xie C, et al: Genome-wide screening reveals an EMT molecular network mediated by Sonic Hedgehog-Gli1 signaling in pancreatic cancer cells. PLoS One 7: e43119, 2012.

13. Lim M, Chuong CM and Roy-Burman P: PI3K, Erk signaling in BMP7-induced epithelial-mesenchymal transition (EMT) of PC-3 prostate cancer cells in 2- and 3-dimensional cultures. Horm Cancer 2: 298-309, 2011. 
14. Li Y, Maitah MY, Ahmad A, Kong D, Bao B and Sarkar FH: Targeting the Hedgehog signaling pathway for cancer therapy. Expert Opin Ther Targets 16: 49-66, 2012.

15. Larue L and Bellacosa A: Epithelial-mesenchymal transition in development and cancer: role of phosphatidylinositol 3 kinase/AKT pathways. Oncogene 24: 7443-7454, 2005.

16. Hennessy BT, Smith DL, Ram PT, Lu Y and Mills GB: Exploiting the PI3K/AKT pathway for cancer drug discovery. Nat Rev Drug Discov 4: 988-1004, 2005.

17. Yang Y, Alcaraz AA and Snyder JP: The tubulin-bound conformation of paclitaxel: T-taxol vs "PTX-NY". J Nat Prod 72: 422-429, 2009.

18. Lee KM, Cao D, Itami A, et al: Class III $\beta$-tubulin, a marker of resistance to paclitaxel, is overexpressed in pancreatic ductal adenocarcinoma and intraepithelial neoplasia. Histopathology 51: 539-546, 2007.

19. Poulain L, Lincet H, Duigou F, et al: Acquisition of chemoresistance in a human ovarian carcinoma cell is linked to a defect in cell cycle control. Int J Cancer 78: 454-463, 1998.
20. Iseri OD, Kars MD, Arpaci F, Atalay C, Pak I and Gündüz U: Drug resistant MCF-7 cells exhibit epithelial-mesenchymal transition gene expression pattern. Biomed Pharmacother 65: 40-45, 2011.

21. Haslehurst AM, Koti M, Dharsee M, et al: EMT transcription factors snail and slug directly contribute to cisplatin resistance in ovarian cancer. BMC Cancer 12: 91, 2012.

22. Maseki S, Ijichi K, Tanaka $\mathrm{H}$, et al: Acquisition of EMT phenotype in the gefitinib-resistant cells of a head and neck squamous cell carcinoma cell line through Akt/GSK-3 $\beta /$ snail signalling pathway. Br J Cancer 106: 1196-1204, 2012.

23. Ahmed N, Abubaker K, Findlay J and Quinn M: Epithelial mesenchymal transition and cancer stem cell-like phenotypes facilitate chemoresistance in recurrent ovarian cancer. Curr Cancer Drug Targets 10: 268-278, 2010.

24. Brabletz T, Jung A, Spaderna S, Hlubek F and Kirchner T: Opinion: migrating cancer stem cells - an integrated concept of malignant tumour progression. Nat Rev Cancer 5: 744-749, 2005. 